\title{
BMJ Open Zinc acetate lozenges for the treatment of the common cold: a randomised controlled trial
}

\author{
Harri Hemilä (D) , ${ }^{1}$ Jari Haukka, ${ }^{1}$ Marianne Alho, ${ }^{2}$ Jussi Vahtera (D) , ${ }^{3,4}$ \\ Mika Kivimäki ${ }^{5,6}$
}

To cite: Hemilä H, Haukka J, Alho $\mathrm{M}$, et al. Zinc acetate lozenges for the treatment of the common cold: a randomised controlled trial. BMJ Open 2020;10:e031662. doi:10.1136/ bmjopen-2019-031662

- Prepublication history and additional material for this paper are available online. To view these files, please visit the journal online (http://dx.doi. org/10.1136/bmjopen-2019031662).

Received 14 May 2019 Revised 29 November 2019 Accepted 04 December 2019

Check for updates

(c) Author(s) (or their employer(s)) 2020. Re-use permitted under CC BY-NC. No commercial re-use. See rights and permissions. Published by BMJ.

${ }^{1}$ Department of Public Health, University of Helsinki, Helsinki, Finland

${ }^{2}$ Espoo City Occupational Health Services, Espoo, Finland

${ }^{3}$ Department of Public Health, University of Turku, Turku,

Finland

${ }^{4}$ Turku University Hospital, Turku,

Finland

${ }^{5}$ Centre of Expertise for Work Organizations, Finnish Institute of Occupational Health, Helsinki, Finland

${ }^{6}$ Department of Epidemiology and Public Health, University College London, London, UK

Correspondence to

Dr Harri Hemilä;

harri.hemila@helsinki.fi

\section{ABSTRACT}

Objective To examine a commercially available zinc acetate lozenge for treating the common cold.

Design Randomised, double-blinded, placebo-controlled trial.

Setting Working population in Finland.

Participants We included men and women aged $\geq 18$ years who usually had $\geq 1$ cold per winter. Exclusions were pregnancy, lactation, chronic runny nose or chronic cough. Intervention We randomised 253 participants to receive a package of lozenges to be taken if they caught the common cold. Of the 253 participants, 88 contracted the common cold and 87 were included in our primary analysis. Zinc acetate lozenges contained $13 \mathrm{mg}$ elemental zinc and placebo lozenges contained sucrose octa-acetate to camouflage the taste of zinc. Instruction to use was six times per day for the maximum of 5 days.

Primary outcome Rate of recovery from the common cold analysed by Cox regression.

Results There was no difference in the recovery rate between zinc and placebo participants during the 10-day follow-up (rate ratio for zinc vs placebo=0.68, 95\% $\mathrm{Cl} 0.42$ to $1.08 ; p=0.10$ ). The recovery rate for the two groups was similar during the 5-day intervention, but for 2 days after the end of zinc/placebo use, the zinc participants recovered significantly slower compared with the placebo participants $(p=0.003)$. In the zinc group, $37 \%$ did not report adverse effects, the corresponding proportion being $69 \%$ in the placebo group.

Conclusions A commercially available zinc acetate lozenge was not effective in treating the common cold when instructed to be used for 5 days after the first symptoms. Taste has been a common problem in previous zinc lozenge trials, but a third of zinc participants did not complain of any adverse effects. More research is needed to evaluate the characteristics of zinc lozenges that may be clinically efficacious before zinc lozenges can be widely promoted for common cold treatment.

Trial registration number NCT03309995.

\section{BACKGROUND}

Several randomised trials have been carried out to test whether zinc lozenges might have treatment effects on the common cold but the findings are mixed. Eight studies have reported significant benefits of zinc lozenges, ${ }^{1-9}$ whereas 12 studies did not find

\section{Strengths and limitations of this study}

- We conducted a preregistered randomised doubleblind placebo-controlled trial to examine a commercially available zinc acetate lozenge for treating naturally occurring common cold episodes.

- We administered zinc lozenges (78 mg/day zinc for 5 days) versus placebo to 253 healthy participants so that they could start treatment with minimal delay after the onset of symptoms; of the 88 who contracted the common cold, 46 received zinc and 42 placebo.

- Due to single dose and duration design, it remains unclear whether the null finding indicates overall lack of efficacy, or too low daily dose or too short duration of the treatment.

- The generalisability of our findings needs to be confirmed in future trials.

benefit ${ }^{9-15}$ one report published six $^{9}$ and another two separate trials, ${ }^{10}$ and one study was published in two separate reports. ${ }^{23}$ Zinc ion can tightly bind to a number of substances, such as citric acid, potentially preventing the release of free zinc ions from lozenges in the oropharyngeal region. Therefore, the formulation of a zinc lozenge is crucial in determining whether a particular lozenge is efficacious. Shortcomings in the formulations and low doses could explain most of the negative findings. ${ }^{16-19}$

Meta-analyses of three zinc acetate studies ${ }^{6-8}$ with a total of 199 patients with common cold found that recovery rate was increased threefold ${ }^{20}$ and colds were shortened on average by 3 days and $36 \% .{ }^{21}$ Zinc gluconate lozenges have been suggested to be less effective than zinc acetate lozenges, ${ }^{16}$ but a meta-analysis of seven trials with a total of 575 common cold participants did not find significant difference between lozenges composed of the two salts. ${ }^{19}$ Zinc doses of up to $200 \mathrm{mg}$ /day have been used, ${ }^{14}$ but a dose-response analysis found that doses over $80-92 \mathrm{mg} /$ day of zinc did not add effectiveness. ${ }^{19}$ 
Zinc lozenges are widely available as over-the-counter medicines for the treatment of colds. Nevertheless, despite certain promising findings, the status of zinc lozenges as a treatment option for the common cold is still uncertain, and they are not currently recommended for the treatment of the common cold in guidelines. ${ }^{22}{ }^{23}$ Although several meta-analyses suggest that zinc lozenges may have the potential to shorten colds, ${ }^{16}{ }^{18-21}$ the findings from trials are heterogeneous indicating that further research is needed to determine the conditions when zinc lozenges are effective and the type and dosage of lozenges that are optimal.

In this preregistered randomised controlled trial, we tested whether the use of a commercially available zinc acetate lozenge with $78 \mathrm{mg}$ /day of elemental zinc, a dose consistent with previously reported positive findings, ${ }^{18} 19$ shortens the duration of the common cold.

\section{METHODS}

\section{Participants}

The randomised controlled trial was conducted from 1 December 2017 to 30 April 2018. Participants were recruited from the employees of City of Helsinki, Finland in collaboration with occupational health unit. We included male and female employees, age $\geq 18$ years and with a self-report that they usually have had $\geq 1$ colds per winter. Exclusion criteria were pregnancy, lactation, chronic runny nose or chronic cough. Participation in the trial did not limit the use of usual healthcare services or over-the-counter medicines during the study.

Initial invitation to the study was sent by email to approximately 6000 employees (figure 1). Of them, 253 met the inclusion and exclusion criteria, were willing to participate, gave signed informed consent and were randomised into treatment (zinc acetate) and comparison (placebo)

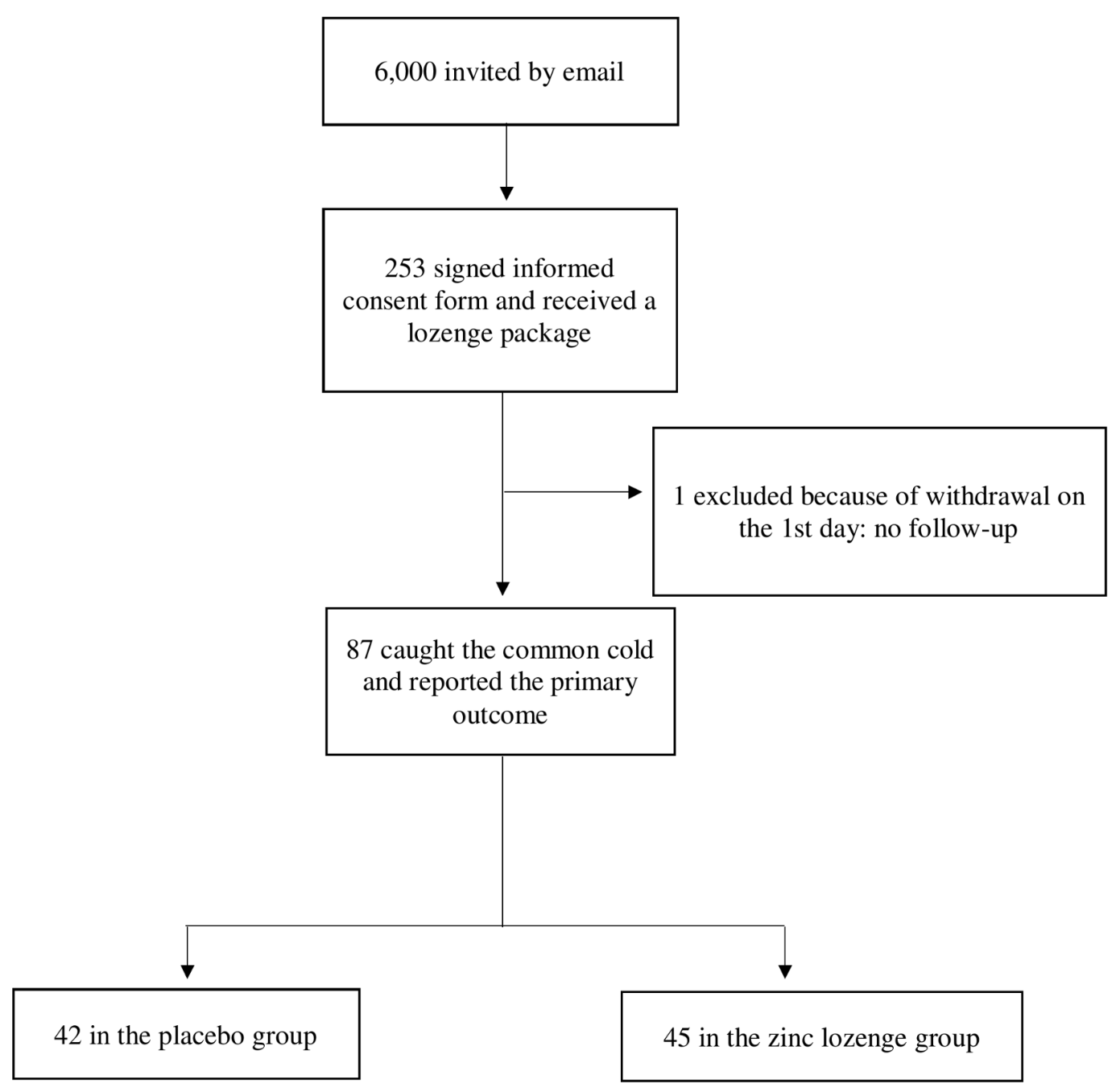

Figure 1 Flowchart of the trial. One participant discontinued the study on the first day and no outcome could be recorded to her; she was administered zinc lozenges. She described that over two decades ago, she had recurrent colds and a few physicians encouraged her to test taking zinc supplements. For one winter, she used zinc and did not suffer from colds, yet over the winter, she started to develop strong adverse reactions from zinc so that she could not continue taking it. Apparently, her strong reaction to the zinc lozenges of our trial were caused by some type of sensitivity generated years earlier. Two of the 45 participants in the zinc group were unable to enter follow-up data through the web-based questionnaire, but we received the primary outcome, the duration of the common cold, by email. 
groups. In total, 88 participants reported common cold during the study period up to 30 April 2018. The final sample of the study was 87 participants (45 in the zinc lozenge group and 42 in the placebo group).

Our trial was registered at the ClinicalTrials.gov with the identifier NCT03309995 (16/10/2017). Our trial was performed in accordance with relevant regulations.

\section{Procedure}

Employees in the chosen work-units of Helsinki City were contacted with an email advertisement in which the criteria for inclusion and exclusion were described and employee's consistency with those criteria was asked. Included in the email was an information sheet, which briefly described the background of the topic. In addition, the email contained a link to a web-based informed-consent form, which was digitally signed by the participants if they decided to participate in the trial.

After signing digitally the informed consent form, the enrolled participants were sent one package of the lozenges. The packages were distributed directly to the participants to allow a more rapid initiation of treatment compared with distributing the lozenges only after the participant actually contracted the common cold. For participants who caught the common cold, we asked baseline data by a web-based questionnaire on the first day of their sickness. The participants were instructed to start taking lozenges as soon as they started to suffer from the first symptoms of the common cold, defined as their personal consideration that they have the common cold, and to start recording their symptoms daily in the webbased questionnaire. The protocol is available at ClinicalTrials.gov and as online supplementary file 1 .

\section{Randomisation and blinding}

The randomisation code was generated at the University Pharmacy and the code was maintained by them until the end of the trial period. The lozenge packages were numbered with 3-digit codes that were used in the identification of the packages (zinc vs placebo). The packages were distributed to participants without the participants and the researchers knowing the contents of the packages. Thus, both participants and researchers were blinded of the type of lozenge during the trial. After the trial was concluded, we asked for the group data as coded to A and $\mathrm{B}$ after cleaning the data and the actual group identification (zinc or placebo) after carrying out the primary analyses of the primary outcome.

\section{Study lozenges}

The zinc lozenge was a commercially available zinc acetate lozenge with $13 \mathrm{mg}$ elemental zinc per lozenge (University Pharmacy, Helsinki, Finland). The lozenge weighed $0.9 \mathrm{~g}$ and had a diameter of $13 \mathrm{~mm}$. The lozenges contained isomaltulose, sorbitol, magnesium stearate, orange and peppermint flavours and sucralose. The instruction of the commercial package for patients with common cold is to dissolve slowly six lozenges per day in the mouth, which totals to $78 \mathrm{mg}$ / day of elemental zinc, at most for 5 days. The same instruction was used in this trial. Each package contained 30 lozenges.

The placebo lozenges, formulated by University Pharmacy, contained $0.13 \mathrm{mg}$ sucrose octa-acetate, which has previously been used in the construction of slightly bitter placebo lozenges to imitate zinc acetate lozenges. ${ }^{6-8}$ The placebo lozenges were closely similar with the zinc lozenges in visual appearance and in taste.

When allowing the zinc and placebo lozenges to slowly dissolve in the mouth, they dissolved in about $8 \mathrm{~min}$.

\section{Outcomes}

On the follow-up period after starting to take the lozenges, using a web-based questionnaire, the participants reported daily about 12 symptoms associated with the common cold: nasal drainage, nasal congestion, sneezing, scratchy throat, sore throat, cough, headache, hoarseness, muscle ache, shivering or feverish, tiredness and difficulty in concentration. These symptoms were asked until the patient recovered or until the 10th follow-up day when the follow-up recording was terminated.

The primary outcome was the self-reported recovery from the common cold, based on the patient's own interpretation when he or she recovered. In the quantification of symptom severity of individual symptoms, we used a scale from 0 to 3 with the following instructions: 0: absent, 1: mild, 2: moderately severe, 3 : very severe. We calculated a total symptom score as the sum over all the symptoms (ie, max 36 points on a day).

In the web-based questionnaire, the patients also reported about adverse effects, about taste and stomach problems and other adverse effects, with an option for free text.

\section{Statistical analyses}

Power calculation: based on an assumption that $60 \%$ of patients with common cold in the zinc acetate group and $30 \%$ in the placebo group would recover by the fifth day, ${ }^{20}$ $42+42$ patients with common cold would be sufficient to reach an $80 \%$ power to detect a difference between the trial groups with $\mathrm{p}<0.05$. In our protocol, we erroneously calculated 23 participants per group as the sample size. Nevertheless, we enrolled participants in their good health and not patients with the common cold. Thus, the number of participants enrolled could not directly translate to an equal number of common cold episodes occurring over the winter in the study groups. For that reason, we sent lozenge packages to 253 participants who had signed the informed consent form (figure 1). The increased sample size allowed for the absence of common cold occurring during the trial winter. It also allowed for a proportion of participants to drop out and some others to have lost their lozenge package.

We used the intention-to-treat approach as far as feasible. In our protocol, we planned to carry out primary analysis by Cox proportional hazards regression over 7 days from the initiation of the intervention. Since there 
was no significant difference between the study groups, we revised the time range and calculated Cox regression over the whole follow-up period of 10 days. The $p$ value for the comparison of the patients still sick on the fourth day and being recovered by the seventh day was calculated with Fisher's exact test. These results are shown in online supplementary file 2. Calculation of the $95 \%$ CI for the rate of no adverse effects was done with the binom.test procedure of $\mathrm{R}^{24}$

All statistical analyses were performed using the $\mathrm{R}$ program package, v.3.4.4. ${ }^{24}$ Statistical significance was determined using 2-tailed $\mathrm{p}<0.05$. The data set of this trial is available as online supplementary file 3 .

\section{Patient and public involvement}

Patients and public were not involved in our formulation of the protocol or in the conduction of the trial. The study findings will be disseminated to the study participants.

\section{RESULTS}

Of the 253 participants who signed informed consent form and were sent a package of lozenges, 88 contracted the common cold during the follow-up from 1 December 2017 to 30 April 2018. One participant had immediately severe adverse effects of the treatment so that she withdrew from the trial on the first day, and we excluded her from the analysis. After opening the code, we found that she was in the zinc group (figure 1). Because of technical problems, two participants were unable to report their symptoms through the web-based questionnaire, but we received the primary outcome (duration of colds) through email.

In our primary analysis, we included 87 participants who contracted the common cold during the follow-up (figure 1). Their characteristics are shown in table 1 . The median age was 49 years, $90 \%$ of the participants were female and $74 \%$ of them had regular contacts with children. A majority of the participants reported having $\geq 2$

\begin{tabular}{|c|c|c|}
\hline & Placebo & Zinc lozenge \\
\hline Total & 42 & 45 \\
\hline Age, mean (SD) & 48 y (9 y) & 46 y $(10 y)$ \\
\hline Males & $3(7 \%)$ & $6(13 \%)$ \\
\hline Contact with children & 32 (76\%) & 32 (71\%) \\
\hline$\geq 2$ colds per winter & $23(55 \%)$ & $24(53 \%)$ \\
\hline $\begin{array}{l}\text { Sick leaves for colds on the } \\
\text { previous winter }\end{array}$ & $29(69 \%)$ & 27 (60\%) \\
\hline Colds usually long & $28(67 \%)$ & $28(62 \%)$ \\
\hline Regular asthma & $4(10 \%)$ & $6(13 \%)$ \\
\hline $\begin{array}{l}\text { Asthma medication for some } \\
\text { previous colds }\end{array}$ & $14(33 \%)$ & $12(27 \%)$ \\
\hline Sinusitis within 5 years & $19(45 \%)$ & $9(20 \%)$ \\
\hline Bronchitis within 5 years & $12(29 \%)$ & $10(22 \%)$ \\
\hline
\end{tabular}

Table 2 Guessing treatment on the first day

\begin{tabular}{lcc}
\hline & Placebo & Zinc lozenge \\
\hline Total & 42 & 43 \\
Guessed placebo & 5 & 7 \\
Guessed zinc & 8 & 10 \\
No guess & 29 & 26 \\
\hline
\end{tabular}

Two participants of the zinc lozenge group are not included since they were unable to fill the web-based questionnaire. We tested if treatment and guessing were independent on lines 2 and $3(p=0.9$ in the $\chi^{2}$ test).

colds per winter, had sick leave on the preceding winter because of the common cold and considered that their colds were usually long. Differences in the baseline characteristics were small between the treatment groups except for the occurrence of sinusitis, which was twice as common in the placebo group as in the zinc lozenge group.

On the first follow-up day, the participants were asked to guess whether they received zinc or placebo lozenges (table 2). Sixty-five per cent of participants did not guess the kind of lozenge that they were taking. Of those who responded either zinc or placebo, about half responded incorrectly, consistent with guessing. Thus, blinding of participants was successful at the start of the trial. On the first day, there was no substantial difference in the symptoms between the study groups with the median of 8 severity points in both the zinc and placebo groups.

The median delay between the start of common cold symptoms and the start of the lozenge treatment was 4 hours, and 31 participants reported that the delay was 2 hours or less. On the second day, $53 \%$ of participants used 6 lozenges and 23\% used five lozenges. Over the period from the second to the fifth days, the zinc participants used on average 5.05 lozenges per day and the placebo participants used 5.15 lozenges per day.

Participants who were administered zinc lozenges recovered on average more slowly than participants administered placebo lozenges (figure 2A). In the Cox regression model over the 10-day follow-up, the zinc lozenge group appeared to recover more slowly although the corresponding rate ratio of 0.68 (95\% CI 0.42 to 1.08; $\mathrm{p}=0.10$ ) was not statistically significant. Given the imbalance in the history of sinusitis between the study groups (table 1), we calculated the effect of zinc lozenges also in participants who did not have sinusitis, and the estimate was little changed. The median duration of colds was 5 days in the placebo group and 7 days in the zinc lozenge group.

The distribution of reported adverse effects is shown in table 3. There was no substantial difference in stomach irritation between the two groups. Taste and other adverse effects were much more common in the zinc lozenge group. Some of the adverse effects classified by participants in the category of 'other' occurred also in the oral region, such as mouth feeling dry or pain in the mouth. 


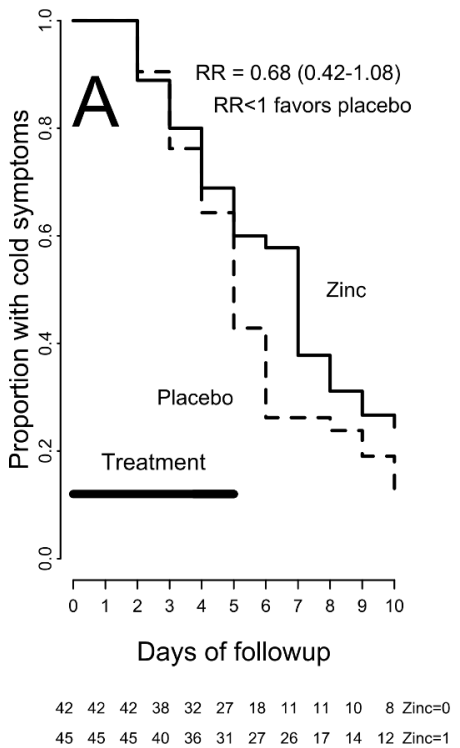

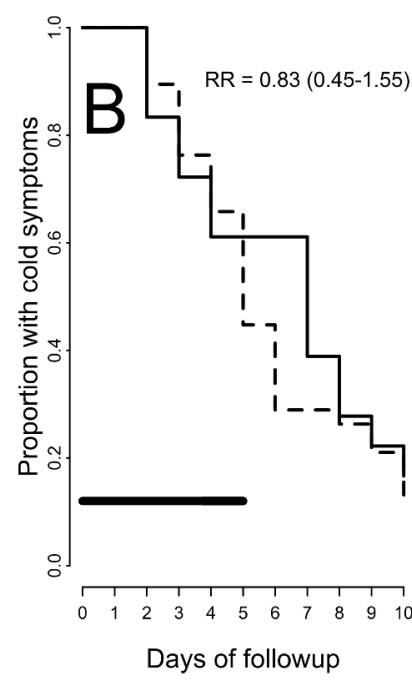

$\begin{array}{lllllllllll}38 & 38 & 38 & 34 & 29 & 25 & 17 & 11 & 11 & 10 & 8 \\ \text { Zinc }=0\end{array}$

$\begin{array}{lllllllllllll}18 & 18 & 18 & 15 & 13 & 11 & 11 & 11 & 7 & 5 & 4 \text { Zinc=1 }\end{array}$

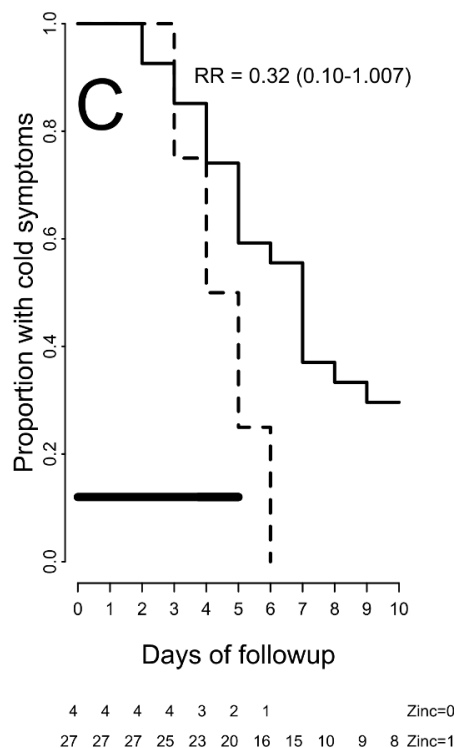

$\begin{array}{llllllllllll}27 & 27 & 27 & 25 & 23 & 20 & 16 & 15 & 10 & 9 & 8 & \text { Zinc }=1\end{array}$

Figure 2 Kaplan-Meier curves describing recovery of participants from the common cold in the zinc lozenge and placebo groups among all participants (A), among 56 participants who did not report any taste or other adverse effects on any of the follow-up recordings $(B)$ and among 31 participants who reported taste or other adverse effects on one or more of their followup recordings (C). In panel $\mathrm{A}$, the recovery rate in the zinc group decreased significantly after the zinc lozenge treatment ended. $R R$, ratio for the recovery rate between zinc and placebo groups, with $R R<1$ indicating that recovery is faster in the placebo group. The number of patients still sick in the two groups is marked at the bottom of the figure. RR, rate ratio.

In the zinc group, only three participants reported other adverse effects without reporting taste adverse effects. Thus, the two categories are largely overlapping and we combined them in table 3 and figure 2B,C. No adverse effects were reported by $37 \%$ ( $95 \%$ CI $23 \%$ to $53 \%$ ) of the zinc lozenge participants. The corresponding proportion was $69 \%$ in the placebo group.

Given the high prevalence of taste and other adverse effects in the zinc group, it seemed possible that the adverse oropharyngeal effects might introduce a bias in reporting the common cold symptoms. For this reason, we carried out an exploratory analysis restricted to 56 participants who did not report any taste or other adverse

\begin{tabular}{|c|c|c|}
\hline & Placebo & Zinc lozenge \\
\hline Total: & 42 & 46 \\
\hline Any adverse effects at any visit & $13(31 \%)$ & $29(63 \%)$ \\
\hline Stomach ache & $11(26 \%)$ & $12(26 \%)$ \\
\hline Taste problems & $3(7 \%)$ & $24(52 \%)$ \\
\hline Other adverse effects* & $1(2 \%)$ & $11(24 \%)$ \\
\hline No adverse effects at any visit & $29(69 \%)$ & $17(37 \%)$ \\
\hline $\begin{array}{l}\text { No taste or 'other' adverse } \\
\text { effects at any visit }\end{array}$ & $38(90 \%)$ & $18(39 \%)$ \\
\hline
\end{tabular}

*Other adverse effects in the zinc group included: teeth and mouth felt dry or rough, aching in the mouth. Thus, many of them were overlapping with taste problems. The participant who withdrew from the trial on the first day is included in this table. For the comparison of any adverse effects at any visit, there is significant difference between the treatment groups with $p=0.003$ in the $\chi^{2}$ test. effects. No difference was seen in the recovery rate between zinc lozenge and placebo groups (figure 2B). A similar analysis of 31 participants who reported taste or other adverse effects found that the recovery rate was greater in the placebo group, with marginal statistical significance (figure 2C).

Among the zinc participants who did not have taste or other adverse effects, there were two consecutive days at the end of the 5-day treatment when no participant recovered from the common cold, suggesting that termination of treatment might have reduced the rate of recovery temporarily (figure 2B). This phenomenon is seen among all the zinc participants as a bulge after the end of the 5-day zinc treatment (figure 2A). On the fourth day, there were 36 participants still sick in the zinc lozenge group and 32 participants in the placebo group (figure 2). Of the participants still sick on the fourth day, $28 \%(10 / 36)$ of the zinc participants recovered by the seventh day, whereas $66 \%(21 / 32)$ of the placebo participants recovered by the seventh day $(\mathrm{p}=0.003)$.

\section{DISCUSSION}

In this randomised controlled trial, we found no evidence that the commercially available zinc acetate lozenge used for a maximum of 5 days is beneficial in treating the common cold. In contrast, the zinc lozenge group seemed to recover on average slightly more slowly than the placebo group, although the difference was not statistically significant at the conventional level.

Our primary outcome was self-reported recovery from the common cold, based on the patient's own interpretation when he or she recovered. Although in ideal 
circumstances a clinical examination would also have been conducted, a more reasonable approach is to use self-diagnosis for clinical research studies of common cold because of the familiarity of subjects with common cold, the variability in symptomatology and lack of rigorous biological definition of the common cold. ${ }^{25}$ It is the person's subjective experience that makes him or her to, for example, meet a physician to ask for a sick leave for the common cold. Patient-reported outcomes that come directly from the patient are also encouraged by the FDA because they are not biased by the interpretations of physicians or anyone else. ${ }^{26}$

We reasoned that the taste problems experienced by a number of participants might have disturbed their sensations in the oropharyngeal region and could thereby confound the reporting of symptoms of the upper respiratory region. Therefore, we carried out an exploratory subgroup analysis in which we found that the divergence between the placebo and zinc lozenge groups was restricted to participants with taste and other adverse effects. This finding is contradictory to the hypothesis by Farr and Gwaltney ${ }^{27}$ that bad taste of zinc lozenges might explain shorter colds in zinc lozenge participants in the very earliest trials. In our zinc group, $56 \%$ reported at least some taste problems and a few of them described that the zinc lozenges tasted terrible; however, taste problems did not lead to a decrease in the average number of lozenges used per day by the zinc participants.
Although our trial with the specific zinc lozenge brand is negative, we do not argue that our results invalidate the eight previous positive reports of zinc lozenges for common cold treatment (see Refs. 1-9; table 4) because various differences in zinc lozenge formulations and our short 5-day intervention can potentially modify the effect. Furthermore, significant benefit of zinc against the common cold has been reported in two trials with nasal zinc gel administration, which lends further support to the concept that local zinc might have therapeutic effects against colds. ${ }^{28}{ }^{29}$ Twelve controlled studies did not find benefit of zinc lozenges, ${ }^{9-15}$ but problems in the lozenge formulations or low doses of zinc cannot be excluded as explanations for the negative findings (see Refs. 16-19; Online supplementary file 2).

Our review of previous literature indicates that $80-92 \mathrm{mg} /$ day of elemental zinc as zinc acetate appears to be an effective dose, ${ }^{18} 19$ and the planned dose in our study was $78 \mathrm{mg}$ /day. In addition, to minimise the delay between the start of cold symptoms and the initiation of treatment, we administered lozenge packages to the homes of the participants. We assumed that this approach might allow a quantitative analysis of the role of delay in the initiation of treatment on the efficacy, compared with requiring a participant to visit a nurse or a physician. At the beginning of our trial, there was no evidence that participants could infer their treatment by looking and tasting their own lozenges. Our sample size calculation

Table 4 Time of dissolving of the lozenges and the dose of zinc in the lozenges

\begin{tabular}{|c|c|c|c|c|c|c|c|c|}
\hline Trial (ref.) & $\begin{array}{l}N(Z n) / N \\
\text { (placebo) }\end{array}$ & $\begin{array}{l}\mathrm{RR}(95 \% \mathrm{Cl}) \text { for the } \\
\text { rate of recovery from } \\
\text { colds }^{*}\end{array}$ & $\begin{array}{l}\text { Weight of } \\
\text { the lozenge } \\
\text { (g) }\end{array}$ & $\begin{array}{l}\text { Zn in the } \\
\text { lozenge } \\
\text { (mg) }\end{array}$ & $\begin{array}{l}\text { Time of } \\
\text { dissolving in } \\
\text { the mouth } \\
\text { (min) }\end{array}$ & $\begin{array}{l}\text { Number of } \mathrm{Zn} \\
\text { lozenges per } \\
\text { dayt }\end{array}$ & $\begin{array}{l}\text { Total Zn } \\
\text { per day } \\
(\mathrm{mg})\end{array}$ & $\begin{array}{l}\text { Total time of } \\
\text { dissolving per day } \\
\text { (hours) }\end{array}$ \\
\hline $\mathrm{Al}-\mathrm{Nakib}^{23}$ & $29 / 28$ & $\S$ & 1.0 & 23 & 20 & 9 & 207 & 3.0 \\
\hline Godfrey $^{420}$ & $35 / 38$ & 1.55 (1.13 to 2.1$)$ & 4.5 & 23.7 & - & 8.1 & 192 & - \\
\hline Mossad $^{520}$ & $49 / 50$ & 2.8 (1.8 to 4.5$)$ & 4.4 & 13.3 & - & 6.0 & 80 & - \\
\hline Prasad $^{7} 20$ & $25 / 23$ & 7.5 (3.5 to 16$)$ & 4.0 & 12.8 & 30 & 6.2 & 80 & 3.1 \\
\hline Prasad $^{80}$ & $25 / 25$ & 22 (7.7 to 64$)$ & 3.8 & 13.3 & 309 & 6.9 & 92 & 3.4 \\
\hline $\begin{array}{l}\text { Turner and } \\
\text { Cetnarowski }\end{array}$ & $69 / 67$ & 1.45 (1.03 to 2.1$)$ & - & 13.3 & - & 6 & 80 & - \\
\hline The current trial & $45 / 42$ & 0.68 (0.40 to 1.14$)$ & 0.9 & 13 & 8 & 5.05 & 65 & 0.7 \\
\hline
\end{tabular}

Comparison of the current zinc lozenge with the zinc lozenges of trials in which zinc lozenges were significantly beneficial in common cold treatment. Five previous trials used zinc gluconate ${ }^{1-59}$ and three used zinc acetate. ${ }^{6-8}$ All studies investigated natural common cold, except Al-Nakib (1987) and Turner (2000), which investigated experimental rhinovirus colds. This table shows the results and dissolving time in eight trials in which zinc lozenges were effective. The potential problems with lozenges in the 12 trials with negative results are described in table $\mathrm{S} 1$ in online supplementary file 2.

${ }^{*}$ The RR calculations are described in Ref. 20 , except for Turner (2000) for which the RR was based on the measurements of the recovery rates from their figure $1 A,{ }^{9}$ see table $\mathrm{S} 2$ in online supplementary file 2 . RR $>1$ indicates that recovery is faster in the zinc lozenge group.

†The number of zinc lozenges per day is either planned ${ }^{129}$ or counted.

$\$ 15 \mathrm{~min}$ is the average for 'dissolved in the mouth as lozenges (about 10-20 min each)'. ${ }^{1}$

$\S$ The Al-Nakib (1987) study ${ }^{23}$ did not report data suitable for the calculation of the RR. The study reported a significant reduction in cold symptoms and in the number of tissues used by the patients, ${ }^{2}$ and a significant effect on the common cold-induced reduction in response time in a psychological test. ${ }^{3}$

IThe dissolving time is based on reporting that the lozenges in Prasad (2008) ${ }^{8}$ were similar to those in Prasad (2000). ${ }^{7}$

${ }^{*}$ The Turner and Cetnarowski study ${ }^{9}$ reported that in the experimental rhinovirus colds trial, the median duration of colds was 2.5 days in the zinc group and 3.5 days in the placebo group $(\mathrm{p}=0.035)$. The same report ${ }^{9}$ published four trials with zinc acetate lozenges that had problems in their composition, which can explain the lack of effect (see Ref. 17; online supplementary table S1). A sixth trial in the same report ${ }^{9}$ administered zinc gluconate lozenges (80 mg/day) to patients with natural colds and found no benefit (online supplementary table S1). The size of the lozenge and its dissolving time were not reported. The number of lozenges per day shown in the table is planned frequency, but actual frequency was not reported.

$\mathrm{RR}$, rate ratio. 
was based on the findings of three previous zinc acetate lozenge trials. ${ }^{6-8} 20$ Thus, we expected positive results.

The unexpected null finding suggests that uncertainty in the efficacy of commercially available zinc lozenges and inappropriate understanding of proper instructions for their use remains. In half of the positive trials, the lozenges weighed about $4 \mathrm{~g}$, whereas our lozenge weighed only $0.9 \mathrm{~g}$ (table 4 ). In five previous trials, the dissolving time of the lozenge in the mouth was $15-30 \mathrm{~min}$, and the cumulative time the participant had a lozenge dissolving in mouth was from 2.3 to 3.4 hours per day. In our study, the lozenges dissolved in mouth in just $8 \mathrm{~min}$ and the cumulative time the participant had a lozenge dissolving in the mouth was only 0.7 hours per day. Two of the positive trials did not report how long their lozenge dissolved in the mouth; however, their lozenges weighed over 4 $\mathrm{g},{ }^{45}$ and it seems evident that they dissolved much more slowly than our $0.9 \mathrm{~g}$ lozenges. In addition, in one of the two trials, ${ }^{4}$ participants used 8.1 lozenges daily, which also increases the cumulative time of dissolving the lozenge compared with the average of 5.1 lozenges in our study.

Although we had recognised that the lozenges in our study were small and dissolved quite rapidly, we did not anticipate that the small size might render these lozenges inactive. Instead, we assumed that the particularly rapid initiation of treatment in our study, with half of the patients starting treatment within 4 hours after the start of common cold symptoms, would provide compensation even if the small size of the lozenge might not be ideal in terms of efficacy. Furthermore, the rapid dissolution of lozenges may have exacerbated taste and other adverse effects. If $80 \mathrm{mg} /$ day of zinc is dissolved in mouth over 0.7 hours per day, the temporary zinc ion concentrations in the oropharyngeal region are several times higher compared with the same zinc dose being dissolved over some 3 hours per day. Evidently, further trials on zinc lozenges should compare the effectiveness between lozenges with various dissolving times.

We considered that the 5-day treatment should be sufficient to demonstrate an effect of zinc acetate lozenges, since in a meta-analysis of three trials with zinc acetate lozenges, a significant benefit of zinc lozenges was seen within 5 days of treatment. ${ }^{20}$ We also assumed that the rapid initiation of zinc lozenges might lead to effects that extend beyond the active treatment period. Nevertheless, the 5-day treatment might have been too short. We found a substantial decrease in the rate of recovery in the zinc participants after the 5-day treatment ended which could be caused by the rebound effect so that the discontinuation of the zinc lozenge treatment had a physiological effect in the harmful direction. Further research is needed to confirm this and it seems evident that further trials on zinc lozenges should not be limited to 5 days of treatment. Some previous studies have administered zinc lozenges for up to 2 weeks. ${ }^{56}$

In our study protocol, we calculated that six lozenges containing $13 \mathrm{mg}$ zinc lead to $78 \mathrm{mg}$ /day of elemental zinc, which is quite consistent with four previous studies in which zinc lozenges were effective..$^{5-8} 1819$ However, we did not take into account that the actual lozenge usages will be lower than planned. On average zinc participants used $65 \mathrm{mg}$ /day of zinc. Although we do not assume that the dose-response between zinc lozenges and their efficacy is such steep that the difference between $65 \mathrm{mg} /$ day and $80-92 \mathrm{mg} /$ day of zinc would explain the inefficacy of our lozenge, further research should examine whether our somewhat low dose is a further factor that led to the negative findings. Apparently, further studies should first use actual doses that are over $92 \mathrm{mg} /$ day and the search for minimal effective dose per day should be carried out only after confirming that a particular formulation is effective.

Bad taste has been a common complaint of zinc lozenges, but there are not much data about the proportion of participants who had no adverse effects. Our observation that $37 \%$ of our zinc participants did not complain of any adverse effects is a substantial proportion. Thus, even if the usefulness of zinc was limited by taste problems, the proportion who are not disturbed by taste may be substantial given that the common cold is a very common ailment.

Eby commented that the majority of zinc lozenges on the US market in 2008 were expected to be ineffective against colds. ${ }^{16}$ Most of the zinc lozenges he surveyed contained citric acid, which binds zinc ions, and many lozenges had such low doses of zinc that they were unlikely to have any pharmacological effects. Thus, although there is evidence from several trials that properly composed zinc lozenges may shorten the duration of colds, ${ }^{1-9}$ a patient with ordinary common cold cannot easily materialise the benefit by zinc lozenges available from a drugstore, a problem further supported by our findings on the 5-day zinc acetate treatment.

\section{Strengths and limitations}

The strengths of this trial include the use of a preregistered protocol and randomised, double-blinded, placebocontrolled design, both contributing to internal validity. Our focus on a widely used commercially available zinc product supports the public health relevance of this study. Since we distributed the lozenges to participants when they were in good health, it was possible to minimise the delay between the onset of symptoms and the start of intervention. There are also some limitations. The assessment of the common cold relied purely on participant's response to a questionnaire while in ideal circumstances a clinical examination would also have been conducted in parallel with the questionnaire. Participants were also not asked to bring lozenge packs with them to a clinic visit for evaluation of compliance based on a pill count, to corroborate their responses to the web questionnaire. Because of the randomised controlled trial design, the subjects were not representative of the general population as we examined efficacy rather than effectiveness of zinc acetate lozenge treatment. 


\section{CONCLUSION}

We found that common cold symptoms were not shortened by a commercially available zinc acetate lozenges with an instruction of $78 \mathrm{mg} /$ day for 5 days. In future trials of the benefits and harms of commercially available zinc lozenges for common cold treatment, the lozenges should be more slowly dissolving in mouth, the dose should be over $92 \mathrm{mg} /$ day and the treatment should last longer than 5 days. The characteristics of zinc lozenges that are suggested to be clinically efficacious should be evaluated in detail before they can be widely promoted for common cold treatment.

Contributors $\mathrm{HH}$ conceived and designed the study, coordinated the trial, prepared the first draft of the manuscript and was responsible for the final manuscript. $\mathrm{HH}, \mathrm{JH}, \mathrm{MA}, \mathrm{JV}$ and MK developed the protocol. HH and MA recruited the patients and acquired the data. $\mathrm{HH}$ did the statistical analysis. $\mathrm{HH}, \mathrm{JH}$ and MK analysed and interpreted the data. All authors revised manuscript drafts, approved the final manuscript and contributed intellectually important content. $\mathrm{HH}$ is the guarantor of the paper and takes responsibility for the integrity of the work as a whole, from inception to published article.

Funding This study was investigator-initiated trial, which was supported by NordForsk (75021) and Academy of Finland (311492). Zinc and placebo lozenges were donated by the University Pharmacy, Helsinki, Finland.

Disclaimer However, University Pharmacy had no role in planning and conducting the trial or in the interpretation of findings and the writing of the manuscript.

Competing interests None declared.

Patient consent for publication Not required.

Ethics approval The Ethics Committee of Helsinki University Central Hospital approved the trial (HUS/2439/2017).

Provenance and peer review Not commissioned; externally peer reviewed.

Data availability statement All data relevant to the study are included in the article or uploaded as supplementary information.

Open access This is an open access article distributed in accordance with the Creative Commons Attribution Non Commercial (CC BY-NC 4.0) license, which permits others to distribute, remix, adapt, build upon this work non-commercially, and license their derivative works on different terms, provided the original work is properly cited, appropriate credit is given, any changes made indicated, and the use is non-commercial. See: http://creativecommons.org/licenses/by-nc/4.0/.

\section{ORCID iDs}

Harri Hemilä http://orcid.org/0000-0002-4710-307X

Jussi Vahtera http://orcid.org/0000-0002-6036-061X

\section{REFERENCES}

1 Eby GA, Davis DR, Halcomb WW. Reduction in duration of common colds by zinc gluconate lozenges in a double-blind study. Antimicrob Agents Chemother 1984;25:20-4.

2 Al-Nakib W, Higgins PG, Barrow I, et al. Prophylaxis and treatment of rhinovirus colds with zinc gluconate lozenges. J Antimicrob Chemother 1987;20:893-901.

3 Smith AP, Tyrrell DA, Al-Nakib W, et al. Effects of zinc gluconate and nedocromil sodium on performance deficits produced by the common cold. J Psychopharmacol 1991;5:251-4.

4 Godfrey JC, Sloane BC, Smith DS, et al. Zinc gluconate and the common cold: a controlled clinical study. J Int Med Res 1992;20:234-46.

5 Mossad SB, Macknin ML, Medendorp SV, et al. Zinc gluconate lozenges for treating the common cold. A randomized, double-blind, placebo-controlled study. Ann Intern Med 1996;125:81-8.

6 Petrus EJ, Lawson KA, Bucci LR, et al. Randomized, doublemasked, placebo-controlled clinical study of the effectiveness of zinc acetate lozenges on common cold symptoms in allergy-tested subjects. Curr Ther Res 1998;59:595-607.

7 Prasad AS, Fitzgerald JT, Bao B, et al. Duration of symptoms and plasma cytokine levels in patients with the common cold treated with zinc acetate. A randomized, double-blind, placebo-controlled trial. Ann Intern Med 2000;133:245-52.

8 Prasad AS, Beck FWJ, Bao B, et al. Duration and severity of symptoms and levels of plasma interleukin-1 receptor antagonist, soluble tumor necrosis factor receptor, and adhesion molecules in patients with common cold treated with zinc acetate. J Infect Dis 2008;197:795-802.

9 Turner RB, Cetnarowski WE. Effect of treatment with zinc gluconate or zinc acetate on experimental and natural colds. Clin Infect Dis 2000;31:1202-8.

10 Farr BM, Conner EM, Betts RF, et al. Two randomized controlled trials of zinc gluconate lozenge therapy of experimentally induced rhinovirus colds. Antimicrob Agents Chemother 1987;31:1183-7.

11 Douglas RM, Miles HB, Moore BW, et al. Failure of effervescent zinc acetate lozenges to alter the course of upper respiratory tract infections in Australian adults. Antimicrob Agents Chemother 1987;31:1263-5.

12 Smith DS, Helzner EC, Nuttall CE, et al. Failure of zinc gluconate in treatment of acute upper respiratory tract infections. Antimicrob Agents Chemother 1989;33:646-8.

13 Weismann K, Jakobsen JP, Weismann JE, et al. Zinc gluconate lozenges for common cold. A double-blind clinical trial. Dan Med Bull 1990;37:279-81.

14 Macknin ML, Piedmonte M, Calendine C, et al. Zinc gluconate lozenges for treating the common cold in children: a randomized controlled trial. JAMA 1998;279:1962-7.

15 Eby GA, Halcomb WW. Ineffectiveness of zinc gluconate nasal spray and zinc orotate lozenges in common-cold treatment: a doubleblind, placebo-controlled clinical trial. Altern Ther Health Med 2006;12:34-8.

16 Eby GA. Zinc lozenges as cure for the common cold - a review and hypothesis. Med Hypotheses 2010;74:482-92.

17 Eby GA. Elimination of efficacy by additives in zinc acetate lozenges for common colds. Clin Infect Dis 2001;32:1520.

18 Hemilä H. Zinc lozenges may shorten the duration of colds: a systematic review. Open Respir Med J 2011;5:51-8.

19 Hemilä $\mathrm{H}$. Zinc lozenges and the common cold: a meta-analysis comparing zinc acetate and zinc gluconate, and the role of zinc dosage. JRSM Open 2017;8:205427041769429.

20 Hemilä H, Fitzgerald JT, Petrus EJ, et al. Zinc acetate lozenges may improve the recovery rate of common cold patients: an individual patient data meta-analysis. Open Forum Infect Dis 2017;4:ofx059.

21 Hemilä H, Petrus EJ, Fitzgerald JT, et al. Zinc acetate lozenges for treating the common cold: an individual patient data meta-analysis. Br J Clin Pharmacol 2016;82:1393-8.

22 Malesker MA, Callahan-Lyon P, Ireland B, et al. Pharmacologic and nonpharmacologic treatment for acute cough associated with the common cold. Chest 2017;152:1021-37.

23 Sexton DJ, McClain MT. The common cold in adults: treatment and prevention. Hirsch MS, Aronson MD, Melin JA, (eds). UptoDate, 2018. Available: https://www.uptodate.com/contents/the-commoncold-in-adults-treatment-and-prevention [Accessed 28 Oct 2018].

24 R Core Team. R: a language and environment for statistical computing. Vienna, Austria: $R$ foundation for statistical computing $(R$ version 3.4.4). Available: https://www.R-project.org/ [Accessed 3 Sep 2019].

25 Eccles R. Is the common cold a clinical entity or a cultural concept? Rhinology 2013;51:3-8.

26 US department of health and human services FDA center for drug evaluation and research, US department of health and human services FDA center for biologics evaluation and research, US department of health and human services FDA center for devices and radiological health. guidance for industry: patient-reported outcome measures: use in medical product development to support labeling claims: draft guidance. Health Qual Life Outcomes 2006;4:79.

27 Farr BM, Gwaltney JM. The problems of taste in placebo matching: an evaluation of zinc gluconate for the common cold. J Chronic Dis 1987;40:875-9.

28 Hirt M, Nobel S, Barron E. Zinc nasal gel for the treatment of common cold symptoms: a double-blind, placebo-controlled trial. Ear Nose Throat J 2000;79:778-82.

29 Mossad SB. Effect of zincum gluconicum nasal gel on the duration and symptom severity of the common cold in otherwise healthy adults. QJM 2003;96:35-43. 